\title{
Solving Resource-Constrained Project Scheduling Problems using Tabu Search
}

\author{
A. Ikonomou ${ }^{1}$, J.E. Galletly ${ }^{2}$ and R.C. Daniel ${ }^{1}$ \\ ${ }^{I}$ University of Buckingham, Buckingham MK18 IEG \\ ${ }^{2}$ The American University in Bulgaria, 2700 Blagoevgrad, \\ Bulgaria (E-mail: JGalletly@nws.aubg.bg)
}

\begin{abstract}
Resource-constrained project scheduling problems are amongst the most difficult combinatorial optimization problems to solve. In this paper, we discuss a project scheduling problem which is labour resource-constrained and includes minimal and maximal buffer times between activities. These lower and upper bounds determine how long an activity has to wait before it can start after a previous activity has finished, and how long it is allowed to wait before it has to start. The values of these buffer times are sequence dependent. The objective is to schedule all activities in order to minimise the makespan, subject to labour resource and buffer constraints.

Tabu Search, one of the newer adaptive heuristics for optimization which has shown remarkable results for other scheduling problems, will be used in order to attempt to find a "good"' solution to this problem. The application of Tabu Search to such a labour resource-constrained project scheduling problem, with minimal and maximal buffer times, is novel.
\end{abstract}

\section{Keywords}

Resource-constrained project scheduling; tabu search; heuristic optimisation

1 INTRODUCTION

\subsection{Project Scheduling}

Project scheduling problems are a generalization of production scheduling problems (Baker, 1974). Such problems are among the hardest combinatorial

The original version of this chapter was revised: The copyright line was incorrect. This has been corrected. The Erratum to this chapter is available at DOI: 10.1007/978-0-387-35390-6_58 
problems to solve with respect to the time and computer power required in order to arrive at an optimal solution. They belong to the class of NP-complete problems.

A project consists of a number of activities. Each activity is processed for a certain amount of time. While processing an activity, any resources necessary to execute this activity have to be allocated to it. Precedence relations define the dependencies between the activities of a project - some activities have to be executed in a certain order, forming chains of activities (Baar et al., 1997). Chains of activities which are not affected by the same precedence relations may be executed concurrently. In general, the constraints on project scheduling problems are determined by the availability restrictions on the resources and the presence of precedence relations.

There are various levels of project scheduling of varying complexity. Classical project scheduling deals only with the time aspect of a project (Içmeli et al., 1993). It does not consider any restrictions with respect to resources (Davis, 1973), (Içmeli et al., 1993), (Herroelen, 1972). However, real-life situations do not correspond to this classical view. Project scheduling in real-life is subject to the availability of certain resources which are required by the activities, but which are available only in limited amounts. The amounts usually are not sufficient to satisfy all the demands of concurrent activities (Davis, 1973). Thus, activities that can process in parallel have to compete against each other for access to these resources (Özdamar and Ulusoy, 1995). The presence of precedence relations and resource limitations increases the difficulty of project scheduling. This level of project scheduling is called a RCPS problem (Davis, 1973), (Herroelen, 1972), (Özdamar and Ulusoy, 1995), and, as a representative of project scheduling, belongs to the class of combinatorial optimization problems.

To find an optimal solution for a specific objective under consideration, the allocation of the scarce resources has to be done in such a way that all precedence restrictions are maintained and all resource requirements are satisfied. Objectives can be time-based, such as minimise project duration or mean lateness, or they can be cost-based, like maximise the project's net present value (NPV) or minimise total costs.

Project scheduling problems have been the subject of extensive research (Içmeli et al., 1993), (Özdamar and Ulusoy, 1995). In recent years, new, potentially encouraging, heuristic, optimisation techniques have been devised. These techniques include Genetic Algorithms (Goldberg, 1989), Tabu Search (Glover, 1989), Simulated Annealing (Kirkpatrick et al., 1983) and Artificial Neural Networks (Hertz et al., 1991).

\subsection{Tabu Search}

Tabu Search (TS) was pioneered by Glover (Glover, 1986) and later developed into a general framework (Glover, 1989), (Glover, 1990), (Glover et al., 1993) TS is a neighborhood search heuristic which incorporates adaptive memory and responsive exploration (Glover, 1994). From an initial starting solution, TS moves 
to neighboring solutions by applying moves to the current solution in order to progessively find a better solution overall, i.e. the (near) global optimum. TS is capable of escaping local optima by using adaptive memory structures, either as short-term memory and/or long-term memory. For example, short-term memory structures (the so-called tabu list) record the moves which are executed in the transition from the current solution to a neighboring solution, and refuse to allow an execution of a move again if the so-called tabu tenure is still active, i.e. if the number of moves, since the particular move was last executed, is less than a value known as the tabu tenure. Such a scheme tries to prevent the search process from entering an endless loop of moves from which it cannot escape. However, it is sometimes useful to overrule the tabu tenure. Aspiration criteria enable the search process to execute a tabu move if no other move is available. A simple aspiration criterion is to overrule the tabu tenure if the resulting solution would yield the best solution found so far in the whole process.

\subsection{Project Scheduling and Tabu Search}

TS has been used on a wide spectrum of problem areas ranging from production/project scheduling to layout and circuit design, and telecommunications to graph problems (Glover and Laguna, 1993). Its application to combinatorial optimization problems has achieved exceptional results (Gendreau et al., 1994), (Barnes and Carlton, 1995), (Malek et al., 1989), (Ikonomou et al., 1996). The application of TS methods to project scheduling is not as widespread as it is for production scheduling.

Project scheduling and, especially, resource-constrained project scheduling, has received much attention in the areas of exact and heuristic techniques. Yet, recent surveys in the domain of project scheduling (Içmeli et al., 1993), (Özdamar and Ulusoy, 1995) cite just one single application of the TS approach. However, a search of the literature reveals more.

Içmeli and Erengüç (Içmeli and Erengüç, 1994) applied TS to a RCPS problem with discounted cash flows - a combination of project scheduling and payment scheduling. They investigated the use of two tabu tenure lists and a diversification strategy which implemented a fixed restart approach.

The approach adopted by Lee and Kim (Lee and Kim, 1996) to the problem class was to implement a generic search procedure, but they used only an interchange move.

Thomas and Salhi (Thomas and Salhi, 1995) also applied TS to the RCPS problem. Their approach allowed infeasible solutions, employed a reduced neighbourhood and used integrated intensification and diversification.

An investigation of TS for the resource levelling problem was carried out by Mushi and Ó hÉigeartaigh (Mushi and Ó hÉigeartaigh, 1997). In Mushi and Ó hÉigeartaigh's novel approach, moves were devised which were suitable to the problem type.

Two new TS algorithms are presented in the report by Baar et al. (Baar et al., 1997). Their investigation concentrated on the RCPS problem. The first of these 
algorithms was based on the elimination of critical arcs on a schedule critical path. The second used an enhanced graph representation which, in addition to conjunctions and disjunctions, included so-called parallelity and flexibility relations.

Of special interest is the research carried out by Cavalcante and de Souza (Cavalcante and de Souza, 1997). They investigated a scheduling problem under labour constraints (SPLC) which resembles the problem type presented in this paper. The search process operated on a sequence of activities which represented a full solution. Three greedy heuristics were used to produce a starting solution. The heuristics were the Total Order Duration (TOD), the Remaining Order Duration (ROD), and the Critical Path (CP). The work demonstrated that TS can find solutions to labour resource-constrained project scheduling problems.

The problem presented in this paper is classified as a labour resourceconstrained project scheduling problem, but with the characteristic of minimum and maximum buffer times.

The next sections will describe the problem instances used, the TS approach taken, as well as the progress made so far, and outline further work.

\section{PROBLEM INSTANCE}

The problem instances, which derive from a real-life problem originating in the EU-funded research project PAMIPS (BASF, Université Catholique de Louvain, Dash Associates, University of Buckingham and Parsytec), concern a certain number of activities that need to be scheduled. The activities are linked by precedence relations, where an activity has one or more predecessors and/or one or more successors, in effect, creating activity chains. These relations are fixed and are considered to be "hard" constraints, i.e. no relaxation is possible.

Each activity comprises a number of operations which are non-preemptive, viz. once an activity has begun, it cannot be interrupted and has to finish all its operations in one go. Each operation has individual labour resource and processing time demands, which constitute the production pattern of an activity. This pattern defines how much labour is needed at each specific point during the total processing time of an activity.

There are no buffer times between operations that belong to the same activity. However, there are buffer times between adjacent activities. For each pair of adjacent activities, upper and lower limits for this buffer are given. Additionally, for each job there exists a time window the job has to start in. These earliest and latest starting times are not allowed to be violated, i.e. they are "hard" constraints.

The labour resource constraints imposed are seen as "soft" constraints which may be violated in the beginning of the search process, although penalized by a heavy penalty, and have to be satisfied later on in order to obtain a feasible schedule, i.e. a schedule with no constraint violations at all.

The idea behind the "soft" constraints is that labour resources are not limited but can be accessed at a higher cost if needed. Thus, initial violations are accepted 
but should gradually be eliminated. Furthermore, a main objective is to generate a feasible schedule as stated above. The literature reports that traditional approaches, such as Integer Programming, have major problems in producing feasible solutions for this problem type (de Souza and Wolsey, 1997). Thus, interest has moved to investigating newer, heuristic techniques such as TS.

The problem, then, is to find a (near) optimal feasible solution for the scheduling of these activities, i.e. one that minimises the makespan of the schedule and observes the constraints.

\section{TABU SEARCH APPROACH}

The basis of the TS approach is the generation of a new, better solution from the current solution, the new solution being found from some "neighbourhood" of the solution space surrounding the current one. (A solution, here, is a schedule for the activities. Each solution must be evaluated to determine its makespan, and to check if it observes the constraints or not, i.e. whether it is feasible or infeasible)

Thus, the core components of a TS process are the implementation of the neighborhood and the class of moves. The most commonly used classes of moves in TS applications are the swap move, i.e. exchange the position of two items, and the insert move, i.e. insert an item between two other items (Barnes and Laguna, 1993), (Barnes et al., 1995). However, the moves employed in this approach are different from the standard insert/swap moves, because, normally, such moves would require a change to the sequence of the activities in the schedule. In this problem, however, due to the activity chains established by the precedence relations, the possibility of sequence changes is very limited. Thus, our neighborhood definition recognizes two novel types of moves, the decrease and the increase move.

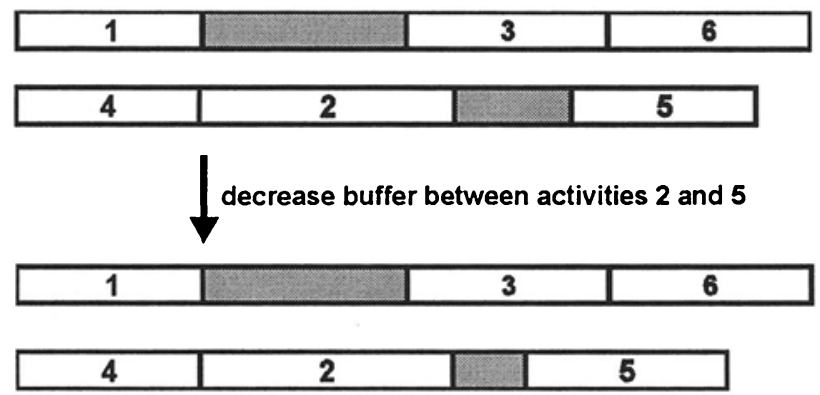

Figure 1 Decrease buffer move

The decrease move decreases the time buffer between two adjacent activities, $i$ and $j$, by one unit, as long as the buffer stays greater than or equal to the minimal buffer allowed between activities $i$ and $j$ (Figure 1 - shading represents a buffer). 


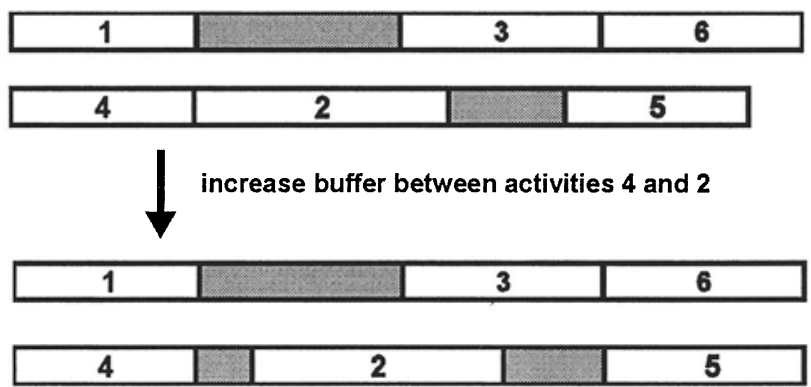

Figure 2 Increase buffer move

The increase move increases the time buffer between activity $i$ and activity $j$ by one unit, again, subject to the adjacency of activity $i$ and activity $j$ in the schedule and in accordance with the buffer restriction, i.e. the buffer will stay less or equal to the maximum buffer allowed (Figure 2).

The neighborhood of the current solution is reached by either decreasing or increasing the buffer between two adjacent activities using these two moves. For a given schedule, there are many combinations of such moves.

With respect to the toleration of infeasible schedules, viz. schedules that violate the labour resource constraints, three attributes are associated with a schedule; firstly, the makespan; secondly, the sum of labour resource violations, i.e. how many more workers have been scheduled than there are available overall; and finally, the cardinality of labour resource violations, i.e. at how many positions in the schedule has a violation actually occurred. The value of an admissible move is determined: by how much it contributes towards the improvement of the schedule with respect to these three attributes.

In order to select the best move generated from the neighbourhood, all admissible moves are ranked. To aid the ranking procedure, three attribute classifications have been introduced. They are the reduction, stagnation, and increase of each respective attribute, e.g. the best move would be one that reduces all three attributes, whereas a move that increases all three attributes would be the worst move possible.

A brief pseudo-code description of the TS method follows:

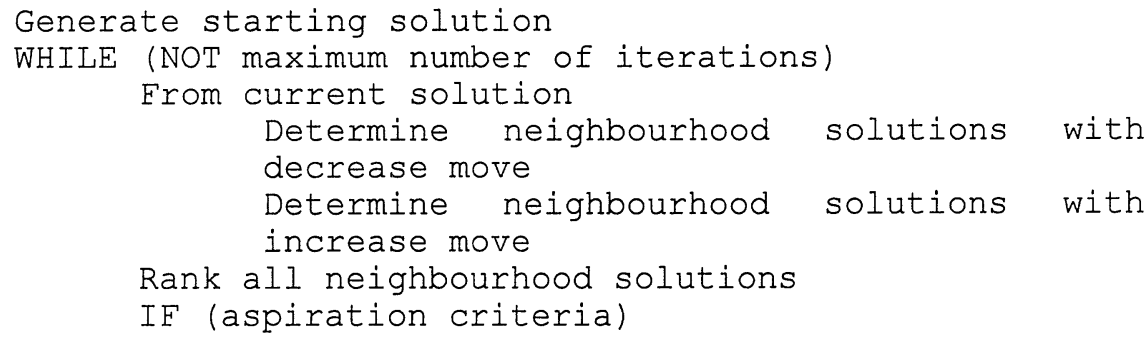




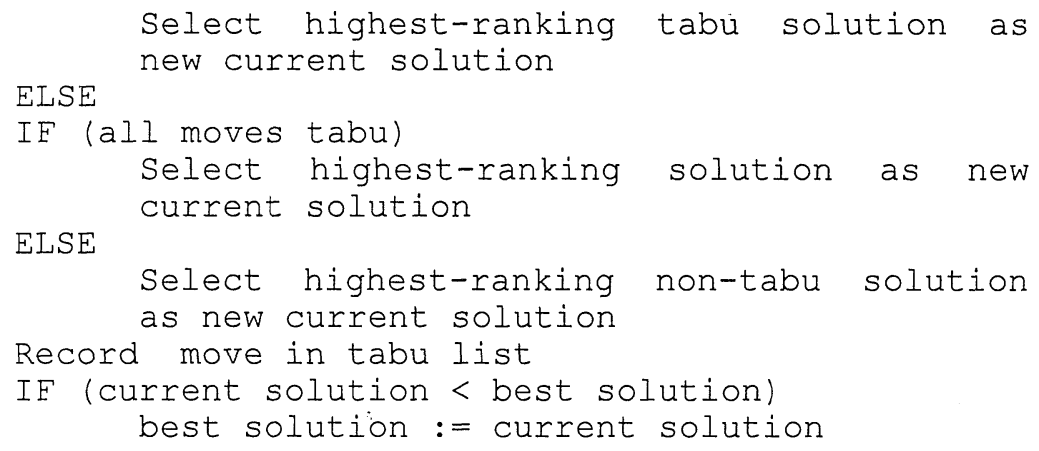

A full schedule representation was designed and used during the TS search process. This schedule contained the schedule length; a number of independent activity sequences (the actual number depends upon the particular problem instance); and the resource availability for each activity.

In order to combat stagnation in the search process, a diversification approach implements a restart strategy and restarts the TS search process alternating between the best infeasible solution and a feasible solution, if available. The restart is invoked when, for example, $5 \%$ of the maximum number of iterations have been executed without an improvement to the feasible solution. Furthermore, diversification is only started after $25 \%$ of the maximum number of iterations has been executed. So, first $25 \%$ of the iterations have to be executed, then the system starts checking for the condition with the 5\%, but only if all moves are tabu. A restart occurs only when the three conditions coincide. These values have been determined empirically. Observations during the experiments lead to the conjecture that the values have to be different for different sizes of problem instances.

In order to generate "good" starting solutions, a modification of a greedy list scheduling algorithm (Dell'Amico and Trubian, 1993) was used. However, the starting solutions produced were allowed to contain partial labour resource infeasibilities. The greedy algorithm has been changed in so far that it schedules the activity which maximizes the makespan first, then all other activities according to the precedence constraints, if present, are scheduled from the back towards the beginning of the schedule by always placing the maximal buffer time between the respective activity pairs. Activities that are not bound by precedence relations are placed in a pool together with the restricted activities. From this pool, the activity with the shortest processing time is chosen to be scheduled next. The starting solution produces a maximum length schedule which can not be increased without violating "hard" constraints, viz. the latest starting times. 
The results presented in this paper represent the initial observations from ongoing research. The testing of the TS algorithm employing the novel moves proceeded in two stages. In the first stage, the TS algorithm performed only one move type, the decrease move, in which the second activity is shifted closer to the first activity in the activity pair, subject to satisfying the minimum and maximum buffer times constraints. The second stage involved the use of both move types, i.e. the decrease move and the increase move and the choice of using a local improvement procedure. Consequently, the results are presented in two parts; results obtained for the single move TS algorithm and results for the complete TS algorithm.

For the first part, small-scale problem instances involving 10, 11 and 12 activities only were investigated. The solution values for this stage are listed in Table 1. (The optimal solutions have been verified by a constraint programming implementation due to Heipcke and Colombani (Heipcke and Colombani, 1997).)

As mentioned in the previously, the first stage of the TS algorithm incorporated only the decrease move. For all three problem instances, the labour resource was set to three units. The problem instances were tested only with this one parameter value because they were intended to confirm the TS algorithm which would then be applied on an extended scope to larger problem instances.

The results showed that, in two out of the three cases, the TS search process did not produce a feasible solution. The 10-activity problem instance returned a best infeasible solution of 21 time units for the makespan, with a total labour deficit of four units, i.e. over-usage. The other problem instance to return an infeasible solution was the 12 -activity problem instance. A makespan of 24 time units with a total labour deficit of four units was the result achieved for this instance, i.e. an over-usage of resources, also. Only for the 11-activity problem instance did the search process produce a feasible solution with the makespan of 19 time units. The globally optimal solution for the 11-activity problem instance is a makespan of 17 units.

Table 1 TS results with only the decrease move implemented

\begin{tabular}{cccc}
$\begin{array}{c}\text { Size } \\
\text { (Number of } \\
\text { Activities) }\end{array}$ & $\begin{array}{c}\text { Labour } \\
\text { Resource } \\
\text { Availability }\end{array}$ & $\begin{array}{c}\text { Best solution found by TS } \\
\text { (Makespan in time units) }\end{array}$ & $\begin{array}{c}\text { Optimum solutions } \\
\text { (Makespan in time } \\
\text { units) }\end{array}$ \\
\hline 10 & 3 & 21 (and infeasible) & 15 \\
11 & 3 & 19 & 17 \\
12 & 3 & 24 (and infeasible) & 21 \\
\hline
\end{tabular}

The inability of the TS algorithm of the first stage to produce feasible solutions was attributed to the fact that only decrease moves were allowed. This 
approach very much resembled a greedy one because the makespan progressively decreased without being offered the chance to increase again. However, this retrograde step would have been necessary if the search was to detect new search trajectories in order to find improved solutions. This effect illustrates the behaviour of greedy algorithms which home in on local optima and cannot escape their attraction. By performing only decrease moves, the search process reached an infeasible local optimum which was remote from any feasible solution region, whether a local or the global optimum one.

The next series of computational experiments was carried out using the second stage of the TS algorithm implementation. The second stage includes both the decrease and the increase move types, and it enables the choice of performing a local optimisation after the generation of the starting solution (although this was not used for the investigations presented here). Additionally, in order to examine the TS algorithm further, both a medium- and large-scale problem instance were included in the test set. These new problem instances had 24 activities with 24 labour resource units, and 88 activities with 27 labour resource units, respectively. The results are summarized in Table 2.

As anticipated, this time the TS implementation yielded feasible solutions for all three small-scale problem instances. The solution value of 15 obtained for 10activity problem instance represents the optimal solution value. The best solution found for the 11-activity problem instance was a makespan of 17 time units which improved the previously found feasible solution by two time units. The solution value is again equal to the globally optimal solution. The initial best solution found for the 12-activity problem instance was a makespan of 21. Again, the global optimum was achieved.

For the medium-scale instance ( 24 activities with 24 labour resource units), the best solution value gave a makespan of 69 time units, a value equal to the global optimum.

Table 2 TS results with both the decrease and increase moves implemented

\begin{tabular}{cccc}
\hline $\begin{array}{c}\text { Size } \\
\text { (Number of } \\
\text { Activities) }\end{array}$ & $\begin{array}{c}\text { Labour } \\
\text { Resource } \\
\text { Availability }\end{array}$ & $\begin{array}{c}\text { Best solution found by TS } \\
\text { (Makespan in time units) }\end{array}$ & $\begin{array}{c}\text { Optimum solutions } \\
\text { (Makespan in time } \\
\text { units) }\end{array}$ \\
\hline 10 & 3 & 15 & 15 \\
11 & 3 & 17 & 17 \\
12 & 3 & 21 & 21 \\
24 & 24 & 69 & 69 \\
88 & 27 & 696 & 399 \\
\hline
\end{tabular}

However, the large-scale instance with 88 activities and 27 labour resource units did not produce a good result. It returned a best solution of 696 time units, compared to the global optimum of 399 . Is there an explanation for the 
performance of the search process seen on this large-scale data set? Certainly for this a problem instance, there is marked increase in intertwined activity chains compared to the others. One possible explanation is the way the search process moves in the neighbourhood of the current solution. The search algorithm employs two move types, the decrease and the increase move. Both moves realise a maximum makespan change of one unit per execution. For a large-scale problem, this might be too small a change to bring the solution value close to the global optimum. It just takes too many moves to reach the 'good' area. Furthermore, the decrease and the increase, in a sense, are in competition. When the decrease moves results in a reduction of the makespan, an increase move might enlarge it again. These circumstances add further to the problem of reaching the area of the global optimum with respect to continuously reducing the makespan.

In conclusion, the computational results with the small- and medium-scale instances have shown that the TS algorithm can solve labour-resource constrained project scheduling problems with minimum and maximum buffer times to optimality, at least for problem instances up to 24 activities and 24 labour resource units. More work is required to understand and improve the result for the large-scale instance.

\section{FURTHER RESEARCH}

The novel move types implemented can be generalised as $k$-decrease and $k$ increase moves where $k$ is equal to one in the current TS implementation. An interesting extension would be to change the value of $k$ and allow the values to range between one and the value of $k$. For example, if $k$ is equal to three then moves which decrease or increase the buffer between two activities for one, two, or three units would be evaluated. This increases the neighborhood size and so appropriate mechanisms would have to be devised which would allow an efficient evaluation of the neighborhood. Probably, a candidate list structure would be a start in this direction.

Furthermore, during the experiments it has been observed that the successor/predecessor checking increases quite considerably with the number of activities in the problem. An approach which tackles this phenomenon in order to reduce the time complexity would be another research area worthwhile investigating.

Additional positive results are expected from the introduction of intensification principles. Intensification will be achieved by always evaluating all possible buffer time settings for an activity pair $i / j$ during one iteration.

Finally, the strategic oscillation concept should be enhanced and refined. One important question to answer in this respect is what exactly is considered feasible/infeasible with respect to activities and labour to qualify for the strategic oscillation concept. How can infeasibilities, in the context of the problem class, be controlled and directed towards feasibility. 
In conclusion, it is envisaged that the final TS implementation will be capable of providing "good" or even optimal solutions to all problem instances of the given resource-constrained project scheduling problem with buffer times. To this end, we have extended our research to cover problem instances for which both the number of activities and labour resource availability are significantly increased (Ikonomou, 1998). We are investigating both medium-scale (20-30 activities) and large-scale (80-90 activities) problem instances, with resource availability varying between 15-30. In many cases, the results are very encouraging.

Baar, T., Brucker, P. and Knust, S. (1997) Tabu-Search Algorithms for the Resource-Constrained Project Scheduling Problem, Fachbereich Mathematik/Informatik, Reihe P Preprints, Heft 192, Universität Osnabrück, Osnabrück, Germany

Baker, K.R. (1997) Introduction to Sequencing and Scheduling. John Wiley, London

Barnes, J. W. and Laguna, M. (1993) A Tabu Search Experience in Production Scheduling. Annals of Operations Research, 41.

Barnes, J. W., Laguna, M. and Glover, F. (1995) An Overview of Tabu Search Approaches to Production Scheduling Problems. Department of Mechanical Engineering, University of Texas at Austin.

Barnes, J. W. and Carlton, W. B: (1995) Solving the Vehicle Routing Problem with Time Windows using Reactive Tabu Search. Department of Mechanical Engineering, University of Texas at Austin.

Cavalcante, C. C. B. and de Souza, C. C. (1997) A Tabu Search Approach for Scheduling Problem Under Labour Constraints. Technical Report IC-97-13, Instituto de Computação, Universidade Estadual de Campinas, Campinas, Brazil.

Davis, E.W. (1973) Project Scheduling under Resource Constraints - Historical Review and Categorization of Procedures. AIIE Transactions, 297-313.

Dell'Amico, M. and Trubian, M. (1993) Applying Tabu Search to the Job-Shop Scheduling Problem, Annals of Operations Research, 41.

Gendreau, M., Hertz, A. and Laporte, G. (1994) A Tabu Search Heuristic for the Vehicle Routing Problem. Management Science, 40, No 10.

Glover, F. (1986) Future Paths for Integer Programming and Links to Artificial Intelligence. Computers and Operations Research, 13, No. 5.

Glover, F. (1989) Tabu Search - Part I, ORSA Journal on Computing, 1, No. 3.

Glover, F. (1990) Tabu Search - Part II, ORSA Journal on Computing, 2, No. 1.

Glover, F., Taillard, E. and deWerra, D. (1993) A user's guide to tabu search. Annals of Operations Research, 41.

Glover, F. and Laguna, M. (1993) Tabu Search, in Modern Heuristic Techniques for Combinatorial Problems,C.R. Reeves ed., Blackwell Scientific Publications. 
Glover, F. (1994) Tabu Search Fundamentals and Uses. Graduate School of Business, University of Colorado at Boulder.

Goldberg, D. E. (1989) Genetic Algorithms in Search Optimization and Machine Learning. Addison-Wesley.

Heipcke, S. and Colombani, Y. (1997) The Constraint Solver SchedEns. Tutorial and Documentation, Technical Report 241, Laboratoire d'Informatique de Marseille, Universite Aix-Marseille II, Marseille, France.

Herroelen, W. S. (1972) Resourcè-constrained Project Scheduling - State of the Art, Operational Research Quarterly, 23, No. 3, 261-75.

Hertz, J., Krogh, A. and Palmer, R. G. (1991) Introduction to the Theory of Neural Computation. Addison-Wesley.

Içmeli, O., Erengüç, S.S. and Zappe, C. J. (1993) Project Scheduling Problems: A Survey. International Journal of Operations and Production Management, 13, No. 11, 80-91.

Içmeli, O. and Erengüç, S.S. (1994) A Tabu Search Procedure for the Resource Constrained Project Scheduling Problem with Discounted Cash Flows. Computers and Operations Research, 21, No. 8, 841-53.

Ikonomou, A., Chua, C., Tan, M., Galletly, J. E. and Daniel R.C. (1996) The Vehicle Routing Problem: A Comparison of a Genetic Algorithm and a Tabu Search Implementation. CO96, Imperial College, London, UK.

Ikonomou, A. (1998) Solving Labour Resource-Constrained Project Scheduling Problems with Minimal and Maximal Buffer Times using Tabu Search, PhD Thesis (in preparation), University of Buckingham, UK

Kirkpatrick, S., Gellat, C. D and Vecchi, M. P. (1983) Optimization by Simulated Annealing. Science, 220.

Lee, J-K. and Kim, Y-D. (1996) Search Heuristics for Resource Constrained Project Scheduling. Journal of the Operational Research Society, 47, No. 5, 678-89.

Malek, M., Guruswamy, M., Pandya, M. and Owens, H. (1989) Serial and Parallel Simulated Annealing and Tabu Search Algorithms for the Traveling Salesman Problem. Annals of Operations Research, 21.

Mushi, A and Ó hÉigeartaigh, M. (1997) The Stochastic Algorithm for the Resource Levelling Problem, Conference of the European Chapter on Combinatorial Optimization (ECCO X), Puerto de la Cruz, Tenerife, Spain.

Özdamar, L., and Ulusoy, G. (1995) A survey on the resource-constrained project scheduling problem. IIE Transactions, 27, 574-86.

de Souza, C.C and Wolsey, L. A. (1997) Scheduling Projects with Labour Constraints. Technical Report IC-97-14, Instituto de Computação, Universidade Estadual de Campinas, Campinas, Brazil.

Thomas, P. R. and Salhi, S. (1995) A Lagrangian Heuristic Approach for the Resource-Constrained Project Scheduling Problem. Working paper 33/95, School of Mathematics and Statistics, University of Birmingham, Birmingham, UK. 